\title{
Microbiological characteristics of municipal solid waste in St. Petersburg
}

\author{
Grigorii Kozlov, and Mikhail Pushkarev ${ }^{1, *}$ \\ ${ }^{1}$ Saint-Petersburg State Institute of Technology, 190013, 26 Moskovsky prospect, St. Petersburg, \\ Russia
}

\begin{abstract}
The article provides information about fluctuations in the microbiological composition of waste from the city of St. Petersburg (Russia) and microbiological air pollution at the facilities for the collection and processing of solid waste. It has been shown that the collection sites do not significantly affect the microbiological pollution of atmospheric air. Large MSW landfills have a significant impact on air pollution by microorganisms. Although the effect of plants for mechanized biological processing of MSW on the microbial number of atmospheric air is observed, it cannot be considered statistically significant.
\end{abstract}

\section{Introduction}

Data on the microbiological composition of municipal solid waste (MSW) are necessary for making managerial decisions in the field of labor protection of personnel and public health at MSW processing enterprises, as well as justification of sanitary protection zones. Waste and compost from waste are reservoirs for the circulation of opportunistic microflora [1-3], which requires continuous monitoring and updating of scientific data. In this regard, it is relevant to conduct research on the microbiological composition of MSW in St. Petersburg by fractions and atmospheric air at the facilities of the waste management system.

\section{Materials and methods}

The studies were carried out using classical techniques of sequential dilutions of the sample and plating on solid diagnostic nutrient media. The main controlled groups of microorganisms were determined by the fractions of MSW. The selection of fractions was carried out after sorting at the site of the enterprise "Mechanized Household Waste Processing Experimental Plant".- a branch of St. Petersburg State Unitary Enterprise Mechanized Household Waste Processing-2

Air sampling was performed by the sedimentation method with recalculation according to the Omelyansko formula. Six petri dishes were used per selection point. Identification of the dominant groups of microorganisms was carried out by the method of MALDI-TOF

\footnotetext{
* Corresponding author:malexpush@bk.ru
} 
mass spectrometry based on the Scientific Research Institute named after Pasteur (St. Petersburg). For mass spectrometric studies, a small number of microorganisms were selected from Petri dishes and applied to the target. In parallel, the same number of microorganisms were applied to the adjacent matrix well and covered with a lysis buffer consisting of $70 \%$ formic acid solution, $30 \%$ acetonitrile solution and $0.1 \%$ sodium dodecyl sulfate (SDS) solution for better destruction of the cell walls and allowed to dry. After the sample dried, $1 \mu \mathrm{l}$ of the matrix ( $\alpha$-cyano-4-hydroxycinnamic acid) was layered on it. Mass spectrometric identification of microorganisms was performed on a MicroFlex mass spectrometer (Bruker, Germany). Each sample was tested in duplicate. Spectra were taken in automatic mode. Standard detection mode - MBT_FC (linear polarization, with a detection range of proteins from 2-20 kDa). 240 spectra were obtained from each sample, which ultimately overlap each other. Identification was performed using the MALDI BioTyper database (version 3.0; if [MSP] 5627 cell microorganisms) (Bruker, Germany). To determine the degree of agreement of the proposed method with the reference, bioinformatic statistics was used - a measure of agreement, which has a maximum of 3.00 with full agreement, and is from 0 to 1,699 if the agreement between the estimates (tests) is observed no more often than might be expected by coincidence, when both indications from 1,700 to 1,999 show approximate results. Values from 2,000 to 2,299 are considered a good degree of agreement, which allows you to accurately determine the type of culture, but the genus of culture does not have a 100\% guarantee of identification. Coefficients from 2,299 to 3,000 show $100 \%$ coincidence of the genus and species of microorganisms.

\section{Results and discussion}

\subsection{The content of microorganisms in the MSW fractions during non-selective waste collection}

The microbiological characteristics of MSW delivered to the MPBO Experimental Plant with gross collection by fractions are given in Tables 1-12 (for months).

Table 1. Microbiological characteristics of MSW St. Petersburg by fractions (January)

\begin{tabular}{|c|c|c|c|c|}
\hline \multirow[t]{2}{*}{ MSW Fraction } & \multicolumn{4}{|c|}{ The content of microorganisms in groups $\mathrm{CFU} / \mathrm{g}$} \\
\hline & 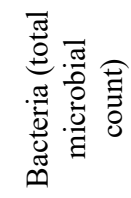 & 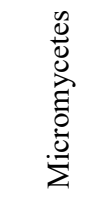 & 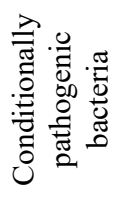 & 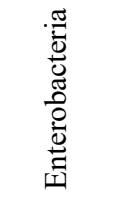 \\
\hline Food waste & $1,0 * 10^{10}$ & $2,0 * 10^{6}$ & $<10^{3}$ & $3,4 * 10^{4}$ \\
\hline Paper & $1,0 * 10^{10}$ & $1,0 * 10^{3}$ & $<10^{3}$ & $<10^{3}$ \\
\hline Wood & $1,0 * 10^{9}$ & $<10^{3}$ & $<10^{3}$ & $<10^{3}$ \\
\hline Black metal & $1,0 * 10^{6}$ & $<10^{3}$ & $<10^{3}$ & $<10^{3}$ \\
\hline Non-ferrous metal & $1,0 * 10^{10}$ & $<10^{3}$ & $2,9 * 10^{3}$ & $2,0 * 10^{4}$ \\
\hline Textile & $1,0 * 10^{10}$ & $<10^{3}$ & $<10^{3}$ & $<10^{3}$ \\
\hline Glass & $6,0 * 10^{10}$ & $2,0 * 10^{7}$ & $8,0 * 10^{4}$ & $5,0^{*} 10^{4}$ \\
\hline Polymers and PET & $1,0 * 10^{6}$ & $<10^{3}$ & $<10^{3}$ & $1,0 * 10^{4}$ \\
\hline Screening & $1,0 * 10^{9}$ & $2,0 * 10^{7}$ & $4,7 * 10^{4}$ & $1,0 * 10^{4}$ \\
\hline Stones, ceramics & $1,0 * 10^{7}$ & $<10^{3}$ & $<10^{3}$ & $<10^{3}$ \\
\hline Other & $1,0 * 10^{9}$ & $3,0 * 10^{3}$ & $<10^{3}$ & $2,0 * 10^{4}$ \\
\hline
\end{tabular}


Table 2. Microbiological characteristics of MSW St. Petersburg by fractions (February)

\begin{tabular}{|c|c|c|c|c|}
\hline \multirow[t]{2}{*}{ MSW Fraction } & \multicolumn{4}{|c|}{ The content of microorganisms in groups CFU / $\mathrm{g}$} \\
\hline & 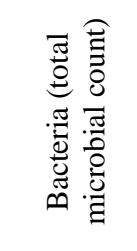 & 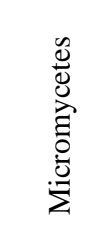 & 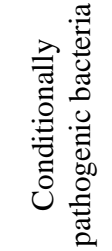 & 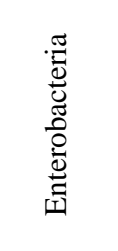 \\
\hline Food waste & $1,0 * 10^{10}$ & $2,0 * 10^{6}$ & $<10^{3}$ & $3,4 * 10^{4}$ \\
\hline Paper & $1,0 * 10^{10}$ & $1,0 * 10^{3}$ & $<10^{3}$ & $<10^{3}$ \\
\hline Wood & $1,0 * 10^{9}$ & $<10^{3}$ & $<10^{3}$ & $<10^{3}$ \\
\hline Black metal & $1,0 * 10^{6}$ & $<10^{3}$ & $<10^{3}$ & $<10^{3}$ \\
\hline Non-ferrous metal & $1,0 * 10^{10}$ & $<10^{3}$ & $2,9 * 10^{3}$ & $2,0 * 10^{4}$ \\
\hline Textile & $1,0 * 10^{10}$ & $<10^{3}$ & $<10^{3}$ & $<10^{3}$ \\
\hline Glass & $6,0 * 10^{10}$ & $2,0 * 10^{7}$ & $8,0 * 10^{4}$ & $5,0 * 10^{4}$ \\
\hline Polymers and PET & $1,0 * 10^{6}$ & $<10^{3}$ & $<10^{3}$ & $1,0 * 10^{4}$ \\
\hline Screening & $1,0 * 10^{9}$ & $2,0 * 10^{7}$ & $4,7 * 10^{4}$ & $1,0 * 10^{4}$ \\
\hline Stones, ceramics & $1,0 * 10^{7}$ & $<10^{3}$ & $<10^{3}$ & $<10^{3}$ \\
\hline Other & $1,0 * 10^{9}$ & $3,0 * 10^{3}$ & $<10^{3}$ & $2,0^{*} 10^{4}$ \\
\hline
\end{tabular}

Table 3. Microbiological characteristics of MSW St. Petersburg by fractions (March)

\begin{tabular}{|c|c|c|c|c|}
\hline \multirow[t]{2}{*}{ MSW Fraction } & \multicolumn{4}{|c|}{ The content of microorganisms in groups CFU / $\mathrm{g}$} \\
\hline & 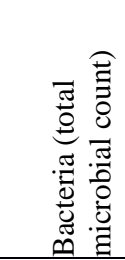 & 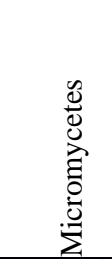 & 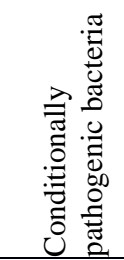 & 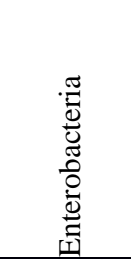 \\
\hline Food waste & $4,0 * 10^{6}$ & $4,0 * 10^{4}$ & $1,0 * 10^{3}$ & $4,1 * 10^{5}$ \\
\hline Paper & $1,0 * 10^{9}$ & $<10^{3}$ & $<10^{3}$ & $1,0 * 10^{5}$ \\
\hline Wood & $1,8 * 10^{9}$ & $<10^{3}$ & $1,0 * 10^{3}$ & $1,0 * 10^{3}$ \\
\hline Black metal & $1,0 * 10^{6}$ & $<10^{3}$ & $<10^{3}$ & $<10^{3}$ \\
\hline Non-ferrous metal & $2,0 * 10^{10}$ & $<10^{3}$ & $<10^{3}$ & $<10^{3}$ \\
\hline Textile & $1,4 * 10^{10}$ & $<10^{3}$ & $2,5 * 10^{4}$ & $1,0 * 10^{5}$ \\
\hline Glass & $2,0 * 10^{5}$ & $<10^{3}$ & $<10^{3}$ & $<10^{3}$ \\
\hline Leather, rubber & $1,2 * 10^{5}$ & $<10^{3}$ & $2,3 * 10^{2}$ & $<10^{3}$ \\
\hline Polymers and PET & $1,0 * 10^{6}$ & $<10^{3}$ & $1,3 * 10^{3}$ & $1,2 * 10^{4}$ \\
\hline Screening & $2,6^{*} 10^{6}$ & $<10^{3}$ & $3,1 * 10^{3}$ & $3,8^{*} 10^{4}$ \\
\hline Stones, ceramics & $6,0 * 10^{5}$ & $<10^{3}$ & $<10^{3}$ & $<10^{3}$ \\
\hline Other & $2,0 * 10^{7}$ & $<10^{3}$ & $7,0^{*} 10^{3}$ & $1,0 * 10^{5}$ \\
\hline
\end{tabular}


Table 4. Microbiological characteristics of MSW of St. Petersburg by fractions (April)

\begin{tabular}{|c|c|c|c|c|}
\hline \multirow[t]{2}{*}{ MSW Fraction } & \multicolumn{4}{|c|}{ The content of microorganisms in groups CFU / $\mathrm{g}$} \\
\hline & 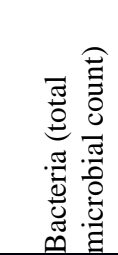 & 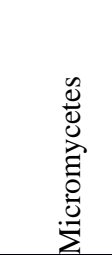 & 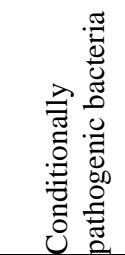 & 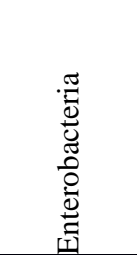 \\
\hline Food waste & $3,1 * 10^{7}$ & $4,0 * 10^{4}$ & $1,0 * 10^{3}$ & $1,0 * 10^{4}$ \\
\hline Paper & $1,0 * 10^{9}$ & $1,0 * 10^{3}$ & $1,0 * 10^{3}$ & $1,0 * 10^{3}$ \\
\hline Wood & $1,0 * 10^{9}$ & $<10^{3}$ & $2,5^{*} 10^{3}$ & $2,7 * 10^{3}$ \\
\hline Black metal & $1,2 * 10^{7}$ & $<10^{3}$ & $<10^{3}$ & $3,2 * 10^{3}$ \\
\hline Non-ferrous metal & $2,0 * 10^{8}$ & $<10^{3}$ & $1,0 * 10^{3}$ & $1,0 * 10^{3}$ \\
\hline Textile & $2,3^{*} 10^{9}$ & $<10^{3}$ & $<10^{3}$ & $<10^{3}$ \\
\hline Glass & $1,0^{*} 10^{7}$ & $2,0 * 10^{3}$ & $1,0 * 10^{3}$ & $1,0 * 10^{3}$ \\
\hline Leather, rubber & $1,2 * 10^{5}$ & $8,0 * 10^{3}$ & $1,3 * 10^{3}$ & $1,0 * 10^{3}$ \\
\hline Polymers and PET & $2,3 * 10^{6}$ & $<10^{3}$ & $1,0 * 10^{3}$ & $1,0 * 10^{3}$ \\
\hline Screening & $3,1 * 10^{6}$ & $1,2 * 10^{4}$ & $1,0 * 10^{4}$ & $1,0 * 10^{4}$ \\
\hline Stones, ceramics & $5,9 * 10^{8}$ & $<10^{3}$ & $<10^{3}$ & $<10^{3}$ \\
\hline Other & $2,0 * 10^{7}$ & $1,0 * 10^{4}$ & $1,0 * 10^{4}$ & $1,0 * 10^{4}$ \\
\hline Food waste & $8,0 * 10^{5}$ & $<10^{3}$ & $1,0 * 10^{4}$ & $1,0 * 10^{3}$ \\
\hline
\end{tabular}

Table 5. Microbiological characteristics of MSW St. Petersburg by fractions (May)

\begin{tabular}{|c|c|c|c|c|}
\hline \multirow[t]{2}{*}{ MSW Fraction } & \multicolumn{4}{|c|}{ The content of microorganisms in groups CFU / g } \\
\hline & 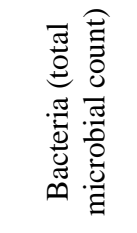 & 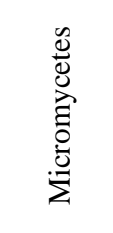 & 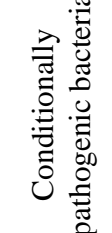 & 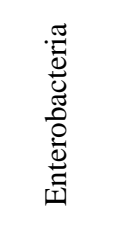 \\
\hline Food waste & $2,0 * 10^{8}$ & $1,0 * 10^{5}$ & $1,0^{*} 10^{4}$ & $1,0 * 10^{4}$ \\
\hline Paper & $1,0 * 10^{8}$ & $<10^{3}$ & $3,0 * 10^{3}$ & $2,0 * 10^{3}$ \\
\hline Wood & $2,0 * 10^{8}$ & $<10^{3}$ & $2,7 * 10^{4}$ & $2,6 * 10^{3}$ \\
\hline Black metal & $3,0 * 10^{7}$ & $<10^{3}$ & $<10^{3}$ & $<10^{3}$ \\
\hline Non-ferrous metal & $1,1 * 10^{11}$ & $<10^{3}$ & $<10^{3}$ & $<10^{3}$ \\
\hline Textile & $3,0 * 10^{9}$ & $<10^{3}$ & $1,0 * 10^{4}$ & $1,0^{*} 10^{4}$ \\
\hline Glass & $1,0 * 10^{9}$ & $3,0 * 10^{4}$ & $4,5 * 10^{4}$ & $1,0 * 10^{4}$ \\
\hline Polymers and PET & $2,0 * 10^{5}$ & $1,4 * 10^{4}$ & $4,1 * 10^{4}$ & $1,0 * 10^{4}$ \\
\hline Screening & $4,0 * 10^{7}$ & $5,0 * 10^{3}$ & $1,5 * 10^{3}$ & $1,0 * 10^{4}$ \\
\hline Stones, ceramics & $2,0 * 10^{8}$ & $<10^{3}$ & $5,8 * 10^{3}$ & $1,0 * 10^{3}$ \\
\hline Leather, rubber & $3,0 * 10^{7}$ & $<10^{3}$ & $4,0 * 10^{3}$ & $1,0 * 10^{3}$ \\
\hline Other & $1,0 * 10^{8}$ & $4,4 * 10^{4}$ & $1,0 * 10^{4}$ & $1,0 * 10^{4}$ \\
\hline Bones & $20 * 10^{7}$ & $<10^{3}$ & $1.0 * 10^{4}$ & $10 * 10^{4}$ \\
\hline
\end{tabular}


Table 6. Microbiological characteristics of MSW St. Petersburg by fractions (June)

\begin{tabular}{|c|c|c|c|c|}
\hline \multirow[t]{2}{*}{ MSW Fraction } & \multicolumn{4}{|c|}{ The content of microorganisms in groups CFU / g } \\
\hline & 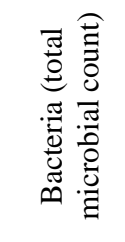 & 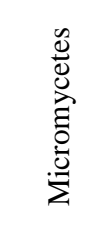 & 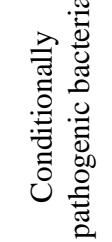 & 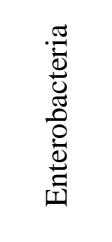 \\
\hline Food waste & $2,0 * 10^{8}$ & $1,0^{*} 10^{5}$ & $1,0 * 10^{5}$ & $1,0 * 10^{5}$ \\
\hline Paper & $1,0 * 10^{8}$ & $<10^{3}$ & $3,4 * 10^{3}$ & $1,0^{*} 10^{4}$ \\
\hline Wood & $1,0 * 10^{8}$ & $2,0^{*} 10^{4}$ & $1,0 * 10^{4}$ & $1,0 * 10^{4}$ \\
\hline Black metal & $1,0 * 10^{6}$ & $<10^{3}$ & $<10^{3}$ & $<10^{3}$ \\
\hline Non-ferrous metal & $1,0 * 10^{7}$ & $1,0 * 10^{3}$ & $1,0 * 10^{3}$ & $1,2 * 10^{3}$ \\
\hline Textile & $6,0 * 10^{8}$ & $2,6 * 10^{5}$ & $1,0 * 10^{5}$ & $1,0 * 10^{5}$ \\
\hline Bones & $1,0 * 10^{7}$ & $2,0^{*} 10^{4}$ & $1,0 * 10^{5}$ & $1,0 * 10^{5}$ \\
\hline Glass & $4,0^{*} 10^{6}$ & $4,0 * 10^{3}$ & $1,0 * 10^{4}$ & $3,7 * 10^{3}$ \\
\hline Leather / rubber & $2,7 * 10^{8}$ & $2,3, * 10^{4}$ & $1,0^{*} 10^{5}$ & $1,0^{*} 10^{5}$ \\
\hline Polymers and PET & $1,0 * 10^{7}$ & $3,0^{*} 10^{4}$ & $4,4 * 10^{4}$ & $1,0^{*} 10^{4}$ \\
\hline Stones / Ceramics & $3,0 * 10^{5}$ & $<10^{3}$ & $8,0 * 10^{3}$ & $2,2 * 10^{3}$ \\
\hline Screening $<15 \mathrm{~mm}$ & $1,0 * 10^{8}$ & $1,0 * 10^{5}$ & $1,0 * 10^{5}$ & $1,0^{*} 10^{5}$ \\
\hline
\end{tabular}

Table 7. Microbiological characteristics of MSW St. Petersburg by fractions (July)

\begin{tabular}{|c|c|c|c|c|}
\hline \multirow[t]{2}{*}{ MSW Fraction } & \multicolumn{4}{|c|}{ The content of microorganisms in groups CFU / $g$} \\
\hline & 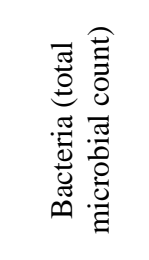 & 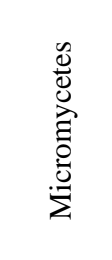 & 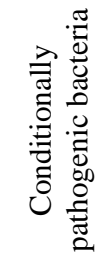 & 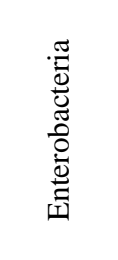 \\
\hline Food waste & $2,0 * 10^{11}$ & $1,6 * 10^{4}$ & $1,0 * 10^{7}$ & $1,0^{*} 10^{6}$ \\
\hline Paper & $1,0 * 10^{11}$ & $1,0 * 10^{4}$ & $<10^{3}$ & $2,0^{*} 10^{7}$ \\
\hline Wood & $2,0 * 10^{11}$ & $<10^{3}$ & $1,0 * 10^{4}$ & $1,6^{*} 10^{7}$ \\
\hline Black metal & $3,0 * 10^{8}$ & $<10^{3}$ & $1,0 * 10^{5}$ & $1,0 * 10^{5}$ \\
\hline Non-ferrous metal & $1,1 * 1010$ & $<10^{3}$ & $8,0 * 10^{3}$ & $1,0 * 10^{6}$ \\
\hline Textile & $3,0 * 10^{11}$ & $2,0 * 10^{3}$ & $7,0 * 10^{3}$ & $1,0 * 10^{5}$ \\
\hline Bones & $2,0 * 10^{9}$ & $2,0 * 10^{3}$ & $1,0 * 10^{6}$ & $1,0 * 10^{6}$ \\
\hline Glass & $1,0 * 10^{8}$ & $<10^{3}$ & $7,0 * 10^{3}$ & $1,0 * 10^{5}$ \\
\hline Leather / rubber & $3,2 * 10^{8}$ & $9 * 10^{3}$ & $1,0 * 10^{4}$ & $1,0^{*} 10^{7}$ \\
\hline Polymers and PET & $2,1 * 10^{8}$ & $1,8 * 10^{4}$ & $1,0 * 10^{6}$ & $1,0^{*} 10^{7}$ \\
\hline Stones / Ceramics & $2,0 * 10^{8}$ & $<10^{3}$ & $5,0 * 10^{3}$ & $1,0^{*} 10^{5}$ \\
\hline Other & $1,0 * 10^{10}$ & $1,8 * 10^{6}$ & $1,0 * 10^{6}$ & $1,0 * 10^{6}$ \\
\hline Screening $<15 \mathrm{~mm}$ & $4,0^{*} 10^{11}$ & $2,8 * 10^{6}$ & $1,0 * 10^{7}$ & $1,0 * 10^{6}$ \\
\hline
\end{tabular}


Table 8. Microbiological characteristics of MSW St. Petersburg by fractions (August)

\begin{tabular}{|c|c|c|c|c|}
\hline \multirow[t]{2}{*}{ MSW Fraction } & \multicolumn{4}{|c|}{ The content of microorganisms in groups CFU / $\mathrm{g}$} \\
\hline & 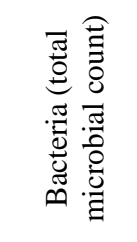 & 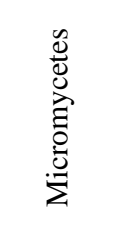 & 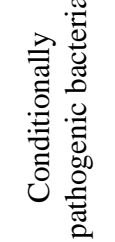 & 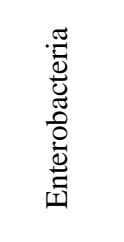 \\
\hline Food waste & $3,1 * 10^{10}$ & $4,1 * 10^{5}$ & $1,0 * 10^{6}$ & $1,0 * 10^{6}$ \\
\hline Paper & $2,3 * 10^{10}$ & $1,0 * 10^{5}$ & $1,0 * 10^{6}$ & $5,0 * 10^{5}$ \\
\hline Wood & $1,0 * 10^{9}$ & $2,0 * 10^{5}$ & $1,0^{*} 10^{6}$ & $1,1 * 10^{5}$ \\
\hline Black metal & $1,0 * 10^{9}$ & $4,2 * 10^{3}$ & $3,1^{*} 10^{4}$ & $1,0 * 10^{5}$ \\
\hline Non-ferrous metal & $1,4 * 10^{9}$ & $<10^{3}$ & $1,0^{*} 10^{4}$ & $1,0 * 10^{6}$ \\
\hline Textile & $1,4 * 10^{10}$ & $1,4 * 10^{4}$ & $8,1^{*} 10^{4}$ & $6,3 * 10^{5}$ \\
\hline Bones & $2,3 * 10^{10}$ & $1,1 * 10^{5}$ & $1,0^{*} 10^{6}$ & $7,1 * 10^{5}$ \\
\hline Glass & $1,0 * 10^{8}$ & $1,3^{*} 10^{5}$ & $1,7 * 10^{5}$ & $1,0 * 10^{5}$ \\
\hline Leather / rubber & $1,2 * 10^{10}$ & $<10^{3}$ & $9,4 * 10^{4}$ & $1,0 * 10^{6}$ \\
\hline Polymers and PET & $2,0 * 10^{8}$ & $1,9 * 10^{5}$ & $1,0 * 10^{5}$ & $2,9 * 10^{5}$ \\
\hline Stones / Ceramics & $2,0 * 10^{10}$ & $1,0 * 10^{4}$ & $1,5 * 105$ & $1,0 * 10^{5}$ \\
\hline Other & $2,8 * 10^{10}$ & $7,1 * 10^{5}$ & $1,0 * 10^{6}$ & $1,0 * 10^{6}$ \\
\hline Screening $<15 \mathrm{~mm}$ & $1,0 * 10^{10}$ & $8,3 * 10^{5}$ & $1,0 * 10^{6}$ & $1,0 * 10^{6}$ \\
\hline
\end{tabular}

Table 9. Microbiological characteristics of MSWSt. Petersburg by fractions (September)

\begin{tabular}{|c|c|c|c|c|}
\hline \multirow[t]{2}{*}{ MSW Fraction } & \multicolumn{4}{|c|}{ The content of microorganisms in groups CFU / $\mathrm{g}$} \\
\hline & 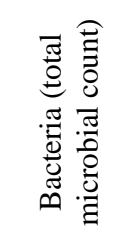 & 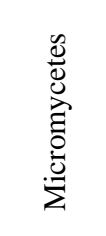 & 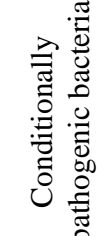 & 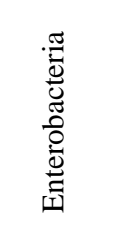 \\
\hline Food waste & $3,0 * 10^{8}$ & $<10^{3}$ & $2,0 * 10^{6}$ & $1,0 * 10^{6}$ \\
\hline Paper & $2,0 * 10^{8}$ & $<10^{3}$ & $<10^{3}$ & $2,4 * 10^{5}$ \\
\hline Wood & $1,0 * 10^{7}$ & $<10^{3}$ & $<10^{3}$ & $1,0 * 10^{3}$ \\
\hline Black metal & $1,0 * 10^{6}$ & $<10^{3}$ & $<10^{3}$ & $1,0 * 10^{5}$ \\
\hline Non-ferrous metal & $1,5^{*} 10^{6}$ & $<10^{3}$ & $1,0^{*} 10^{4}$ & $2,0 * 10^{4}$ \\
\hline Textile & $2,8^{*} 10^{8}$ & $<10^{3}$ & $8,0^{*} 10^{4}$ & $1,3 * 10^{4}$ \\
\hline Glass & $1,2 * 10^{6}$ & $<10^{3}$ & $<10^{3}$ & $<10^{3}$ \\
\hline Leather / rubber & $1,0^{*} 10^{6}$ & $<10^{3}$ & $<10^{3}$ & $<10^{3}$ \\
\hline Polymers and PET & $2,0 * 10^{5}$ & $<10^{3}$ & $<10^{3}$ & $3,0 * 10^{4}$ \\
\hline Stones / Ceramics & $1,0^{*} 10^{6}$ & $<10^{3}$ & $3 * 10^{5}$ & $3,1 * 10^{4}$ \\
\hline Other & $2,6^{*} 10^{6}$ & $1,0 * 10^{5}$ & $1,3 * 10^{6}$ & $1,8 * 10^{6}$ \\
\hline Screening $<15 \mathrm{~mm}$ & $1,0^{*} 10^{8}$ & $1,0 * 10^{5}$ & $<10^{3}$ & $1,0 * 10^{5}$ \\
\hline
\end{tabular}


Table 10. Microbiological characteristics of MSW St. Petersburg by fractions (October)

\begin{tabular}{|c|c|c|c|c|}
\hline \multirow[t]{2}{*}{ MSW Fraction } & \multicolumn{4}{|c|}{ The content of microorganisms in groups CFU / $\mathrm{g}$} \\
\hline & 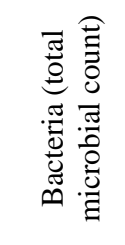 & 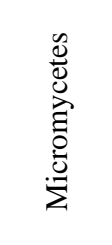 & 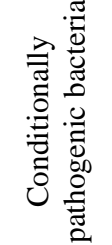 & 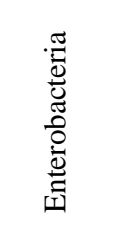 \\
\hline Food waste & $3,0 * 10^{8}$ & $5,4 * 10^{5}$ & $2,1 * 10^{6}$ & $1,0 * 10^{6}$ \\
\hline Paper & $2,0 * 10^{8}$ & $1,0 * 10^{4}$ & $1,0 * 10^{5}$ & $1,9 * 10^{4}$ \\
\hline Wood & $3,0 * 10^{7}$ & $3,0 * 10^{3}$ & $1,9 * 10^{4}$ & $1,0 * 10^{5}$ \\
\hline Non-ferrous metal & $4,0^{*} 10^{7}$ & $<10^{3}$ & $<10^{3}$ & $1,0 * 10^{4}$ \\
\hline Textile & $2,0 * 10^{8}$ & $1,8 * 10^{4}$ & $1,3^{*} 10^{5}$ & $1,3 * 10^{5}$ \\
\hline Bones & $8,1 * 10^{9}$ & $1,5^{*} 10^{4}$ & $2,0 * 10^{6}$ & $4,0 * 10^{4}$ \\
\hline Glass & $1,0^{*} 10^{6}$ & $<10^{3}$ & $1,2 * 10^{4}$ & $2,5 * 10^{4}$ \\
\hline Leather / rubber & $2,0 * 10^{7}$ & $<10^{3}$ & $2,0 * 10^{3}$ & $<10^{3}$ \\
\hline Polymers and PET & $2,0 * 10^{7}$ & $<10^{3}$ & $4,0 * 10^{4}$ & $7 * 10^{4}$ \\
\hline Stones / Ceramics & $3,0^{*} 10^{7}$ & $<10^{3}$ & $6,0^{*} 10^{5}$ & $1,7 * 10^{4}$ \\
\hline Other & $3,0 * 10^{7}$ & $1,6^{*} 10^{5}$ & $1,0 * 10^{6}$ & $1,4 * 10^{6}$ \\
\hline Screening $<15 \mathrm{~mm}$ & $1,0 * 10^{7}$ & $2,3 * 10^{5}$ & $2,9 * 10^{5}$ & $3,4 * 10^{6}$ \\
\hline
\end{tabular}

Table 11. Microbiological characteristics of MSW St. Petersburg by fractions (November)

\begin{tabular}{|c|c|c|c|c|}
\hline \multirow[t]{2}{*}{ MSW Fraction } & \multicolumn{4}{|c|}{ The content of microorganisms in groups CFU / $g$} \\
\hline & 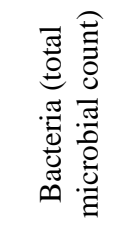 & 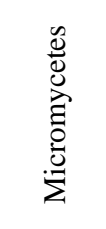 & 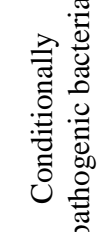 & 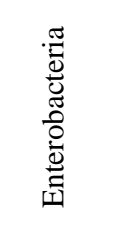 \\
\hline Food waste & $1,0 * 10^{7}$ & $2,0 * 10^{4}$ & $3,7 * 10^{5}$ & $3,2 * 10^{6}$ \\
\hline Paper & $3,0 * 10^{7}$ & $5,0 * 10^{3}$ & $4,0 * 10^{5}$ & $1,2 * 10^{6}$ \\
\hline Wood & $2,0 * 10^{6}$ & $5,0 * 10^{3}$ & $2,0 * 10^{4}$ & $3,0 * 10^{4}$ \\
\hline Black metal & $1,9 * 10^{8}$ & $2,1 * 10^{3}$ & $6,9 * 10^{4}$ & $1,8 * 10^{4}$ \\
\hline Non-ferrous metal & $4,0^{*} 10^{7}$ & $2,0 * 10^{3}$ & $3,1 * 10^{4}$ & $5,0 * 10^{4}$ \\
\hline Textile & $6,0 * 10^{8}$ & $3,0 * 10^{3}$ & $6,0 * 10^{5}$ & $1,0 * 10^{6}$ \\
\hline Bones & $2,0^{*} 10^{8}$ & $2,0 * 10^{4}$ & $6,8 * 10^{6}$ & $2,0 * 10^{5}$ \\
\hline Glass & $2,0 * 10^{6}$ & $1,0 * 10^{3}$ & $3,6^{*} 10^{4}$ & $3,8 * 10^{5}$ \\
\hline Leather / rubber & $1,9 * 10^{8}$ & $<10^{3}$ & $1,0 * 10^{3}$ & $<10^{3}$ \\
\hline Polymers and PET & $5,8^{*} 10^{7}$ & $4,0 * 10^{3}$ & $4,3 * 10^{4}$ & $1,3 * 10^{5}$ \\
\hline Stones / Ceramics & $1,2 * 10^{9}$ & $6,0 * 10^{3}$ & $5,8 * 10^{3}$ & $1,0 * 10^{4}$ \\
\hline Other & $2,0 * 10^{7}$ & $7,1 * 10^{5}$ & $6,7 * 10^{6}$ & $4,1 * 10^{6}$ \\
\hline Screening $<15 \mathrm{~mm}$ & $1,0 * 10^{8}$ & $2,0 * 10^{4}$ & $3,0^{*} * 10^{5}$ & $<10^{3}$ \\
\hline
\end{tabular}


Table 12. Microbiological characteristics of MSW of St. Petersburg by fractions (December)

\begin{tabular}{|c|c|c|c|c|}
\hline \multirow[t]{2}{*}{ MSW Fraction } & \multicolumn{4}{|c|}{ The content of microorganisms in groups CFU / g } \\
\hline & 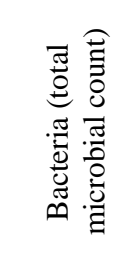 & 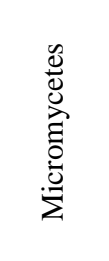 & 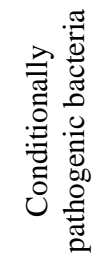 & 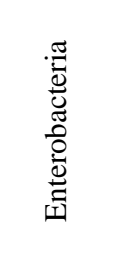 \\
\hline Food waste & $1,0 * 10^{10}$ & $4,0 * 10^{4}$ & $1,9 * 10^{4}$ & $4,1 * 10^{5}$ \\
\hline Paper & $1,0 * 10^{10}$ & $1,0 * 10^{3}$ & $<10^{3}$ & $1,0 * 10^{4}$ \\
\hline Wood & $1,0^{*} 10^{9}$ & $<10^{3}$ & $<10^{3}$ & $2,7 * 10^{3}$ \\
\hline Black metal & $1,0 * 10^{6}$ & $<10^{3}$ & $<10^{3}$ & $3,2 * 10^{3}$ \\
\hline Non-ferrous metal & $1,0 * 10^{10}$ & $<10^{3}$ & $3,0 * 10^{3}$ & $1,0 * 10^{3}$ \\
\hline Textile & $1,0 * 10^{9}$ & $<10^{3}$ & $<10^{3}$ & $1,0 * 10^{5}$ \\
\hline Glass & $1,0 * 10^{9}$ & $2,0 * 10^{7}$ & $<10^{3}$ & $1,0 * 10^{4}$ \\
\hline Polymers and PET & $1,0 * 10^{6}$ & $1,0 * 10^{5}$ & $<10^{3}$ & $1,2 * 10^{3}$ \\
\hline Screening & $1,0 * 10^{9}$ & $2,0 * 10^{7}$ & $4,7 * 10^{4}$ & $<10^{3}$ \\
\hline Stones, ceramics & $1,0 * 10^{6}$ & $<10^{3}$ & $3,0 * 10^{3}$ & $1,0 * 10^{4}$ \\
\hline Leather, rubber & $1.0 * 10^{6}$ & $3,0 * 10^{4}$ & $<10^{3}$ & $1,0 * 10^{5}$ \\
\hline
\end{tabular}

Microorganisms less than $10^{3} \mathrm{CFU} / \mathrm{g}$ are not considered. The total bacterial content in the hygroscopic fractions of MSW (total microbial number, hereinafter referred to as TMP) is greater in the autumn - winter period. The content of fungi, opportunistic and colimorphic microorganisms is more dependent on temperature and minimally in winter and spring. In summer, the OMC fractions are lower due to moisture evaporation, however, high temperatures provide the maximum microflora content related to opportunistic and enterobacteria. In general, solid waste collected by the shaft represent a serious danger to the personnel of waste sorting stations and the surrounding residential development due to the high content of microorganisms. Before manual sorting, it is necessary to separate the fractions of "food waste", "screening" and "other" using a separator.

The seeding of MSW fractions is influenced by a large number of factors, meteorological conditions are one of the main ones (weather conditions at the time of sampling were taken from meteorological sites: temperature, humidity and rainfall (sources: http://pitermeteo.ru, http://www.meteo.nw.ru )). Thus, the pairwise correlation coefficients of the contamination of food waste with the monthly average temperature $(0.120)$ and humidity $(0.222)$ indicate an extremely weak correlation of these values, however, the correlation is more pronounced with the amount of precipitation (0.620). If we calculate the correlation coefficient of precipitation for months with a negative average monthly temperature (0.894), it will be significantly higher than with positive (0.574). For opportunistic and enterobacteria, ambient temperature is more important. In spite of the fact that measurement errors make a significant contribution, since the monthly average hydrometeorological data are used for St. Petersburg as a whole, and not for a specific sampling point, it can be stated that the key factor affecting the microbial number of MSW is the waste moisture, which is summed up as intrinsic humidity brought mainly by food waste and atmospheric precipitation in MSW i.e. essentially a collection system. Separate collection of food waste that existed in Leningrad in the 70-80s of the twentieth century made it possible to reduce the amount of solid waste by 3-4 times. Separate collection of sorted fractions and wet municipal solid waste in combination with sheltering the collection sites from precipitation is the best solution aimed at minimizing the microbiological risks associated with MSW. 


\subsection{The effect of separate collection on the content of microorganisms in the fractions of MSW}

In order to assess the degree of reduction of MSW contamination during separate collection, the effect of separate collection of dry and wet fractions of MSW on their microbiological composition was studied. Samples were taken at the MSW collection stage in households. Investigated the contamination of the fractions in the gross collection of wet waste and separate collection of air-dry waste. The results are shown in tables 13 and 14 .

Table 13. Microbiological characteristics of MSW St. Petersburg by fractions (wet waste, gross yield)

\begin{tabular}{|c|c|c|c|c|c|}
\hline \multirow[b]{2}{*}{ Fraction } & \multirow[b]{2}{*}{ 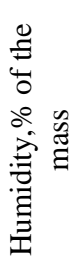 } & \multicolumn{4}{|c|}{ The number of M / O dry weight } \\
\hline & & 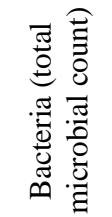 & 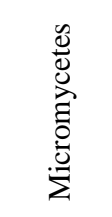 & 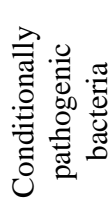 & 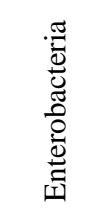 \\
\hline Food waste & 92,8 & $9,5 * 10^{7}$ & $3,4 * 10^{7}$ & $6,8 * 10^{4}$ & $5,7 * 10^{7}$ \\
\hline Paper & 27,5 & $4,2 * 10^{7}$ & $1,6^{*} 10^{6}$ & $2,1 * 10^{7}$ & $1,0 * 10^{7}$ \\
\hline Non-ferrous metal (foil) & 8,3 & $4,1 * 10^{6}$ & $7,1 * 10^{5}$ & $2,9 * 10^{5}$ & $1,7 * 10^{6}$ \\
\hline Textile & 43,8 & $3,0 * 10^{8}$ & $6,0^{*} 10^{7}$ & $6,9 * 10^{7}$ & $7,6^{*} * 10^{7}$ \\
\hline Bones & 67,9 & $9,8 * 10^{6}$ & $<10^{3}$ & $<10^{3}$ & $<10^{3}$ \\
\hline Glass & 0,6 & $<10^{3}$ & $<10^{3}$ & $<10^{3}$ & $<10^{3}$ \\
\hline Leather and rubber & 7,1 & $<10^{3}$ & $<10^{3}$ & $<10^{3}$ & $<10^{3}$ \\
\hline Polymers & 0,1 & $8,6^{*} 10^{6}$ & $2,9 * 10^{4}$ & $5,7 * 10^{5}$ & $2,9 * 10^{5}$ \\
\hline PET & 0,2 & $7,6^{*} 10^{4}$ & $4,0^{*} 10^{4}$ & $4,4 * 10^{4}$ & $8,0 * 10^{4}$ \\
\hline Other & 79,7 & $2,6^{*} 10^{7}$ & $2,6^{*} 10^{7}$ & $2,6 * 10^{7}$ & $2,0 * 10^{7}$ \\
\hline Screening $<15 \mathrm{~mm}$ & 8,6 & $5,3^{*} 10^{7}$ & $1,1 * 10^{7}$ & $1,3 * 10^{8}$ & $2,0 * 10^{7}$ \\
\hline
\end{tabular}

The most seeded components of wet waste are the most hygroscopic fractions of "food waste", "screening" and "other", textiles. It is noteworthy that the "screening" fraction is more than half organic waste [4]. Less hygroscopic fractions ("skin and rubber") are seeded several orders of magnitude lower. In the fractions of air-dry MSW, the microflora content falls by 5-8 orders of magnitude. Thus, humidity is a determining factor that positively affects the content of microorganisms in MSW, however, humidity can also negatively affect the number of microorganisms - thus, the data on the fractionation of the fractions for the month of September (table 9) at first glance completely do not correspond to environmental conditions and morphological composition, however the extremely low content of microorganisms (primarily micromycetes) is explained by excessive humidity, in which the waste is saturated with water and has no voids for aeration and substrate.

It should also be noted that the content of microorganisms in the waste increases during the stay in the container and during transportation in containers to the place of sorting and / or processing.

The species composition of the microflora of MSW fractions is also different in composition (only dominant species isolated on solid nutrient media with minimal dilutions were determined): food waste fraction - Bacillus cereus and Achromobacter denitrificans species dominate; in the fractions "paper", "textiles" - species of Enterobacter cloacae, E. asbuburiae, Stenotrophomonas maltophilia; the glass fraction contains Bacillus thuringiensis; the "other" fraction of the species Klebsiella oxutoca, Pseudomonas sp., Rahnella aquatilis; "Screening" - Geobacillus stearothermophilus, Pseudomonas fulva. Species whose content was less than $10^{3} \mathrm{CFU} / \mathrm{g}$ were not taken into account and were not 
determined. Thermophilic $\left(+55^{\circ} \mathrm{C}\right)$ microorganisms in the composition of MSW fractions are represented by the species Geobacillus stearothermophilus.

Table 14. Microbiological characteristics of MSW St. Petersburg by fractions (air-dry waste, separate collection)

\begin{tabular}{|c|c|c|c|c|c|}
\hline \multirow[b]{2}{*}{ Fraction } & \multirow[b]{2}{*}{ 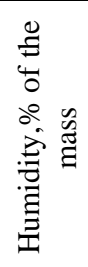 } & \multicolumn{4}{|c|}{ The number of M / O dry weight } \\
\hline & & 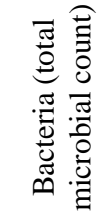 & 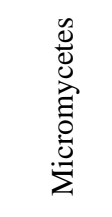 & 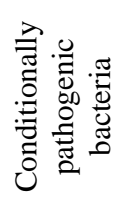 & 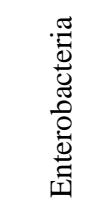 \\
\hline Food waste & 6,7 & $1,2 * 10^{3}$ & $<10^{3}$ & $<10^{3}$ & $<10^{3}$ \\
\hline Paper & 4,2 & $2,3 * 10^{4}$ & $2,3 * 10^{4}$ & $<10^{3}$ & $2,3 * 10^{4}$ \\
\hline Non-ferrous metal (foil) & 0 & $<10^{3}$ & $<10^{3}$ & $<10^{3}$ & $<10^{3}$ \\
\hline Textile & 2,5 & $2,2 * 10^{6}$ & $6,2 * 10^{5}$ & $3,0 * 10^{5}$ & $3,1 * 10^{6}$ \\
\hline Glass & 0,1 & $<10^{3}$ & $<10^{3}$ & $<10^{3}$ & $<10^{3}$ \\
\hline Leather and rubber & 13,3 & $<10^{3}$ & $<10^{3}$ & $<10^{3}$ & $<10^{3}$ \\
\hline Polymers & 0 & $1,5 * 10^{3}$ & $1,2 * 10^{3}$ & $<10^{3}$ & $<10^{3}$ \\
\hline PET & 0 & $2,9 * 10^{3}$ & $5,7 * 10^{3}$ & $<10^{3}$ & $<10^{3}$ \\
\hline Other & 4,8 & $7,0 * 10^{5}$ & $2,3 * 10^{6}$ & $2,2 * 10^{6}$ & $1,7 * 10^{6}$ \\
\hline Screening $<15 \mathrm{~mm}$ & 5,0 & $6,8 * 10^{4}$ & $3,7 * 10^{4}$ & $2,5^{*} 10^{3}$ & $5,2 * 10^{4}$ \\
\hline
\end{tabular}

\subsection{The impact of waste management facilities on microbiological air pollution}

Air samples were taken in various regions of St. Petersburg. Six Petri dishes were used for one collection point (Figure 1.) The results of the study are shown in Table 15. The content of microorganisms in the air at the MSW collection sites does not differ from that for the air in the studied area of St. Petersburg. So in the control samples of the Petrogradsky district location - bacteria are $(1.89 \pm 0.09) \times 10^{4}$, micromycetes $(4.51 \pm 0.41) \times 10^{3}$; in the location Kalininsky district - bacteria $(7.46 \pm 0.10) \times 10^{3}$, micromycetes $(2.12 \pm 0.33) \times 10^{3}$; in the location of the Kirovsky district $(2.56 \pm 0.69) \times 10^{3},(1.21 \pm 0.23) \times 10^{3}$, respectively.

In the territory of the garbage processing plant and landfill, microbiological contamination (table 16) significantly exceeds that for the nearest residential development taken as a control, thus, MSW collection sites do not negatively affect the microbiological composition of the air, and landfills significantly affect the microbiological pollution of the atmosphere. Mechanized waste processing plants influence atmospheric pollution, but it cannot be considered reliable, since confidence intervals of values overlap. 


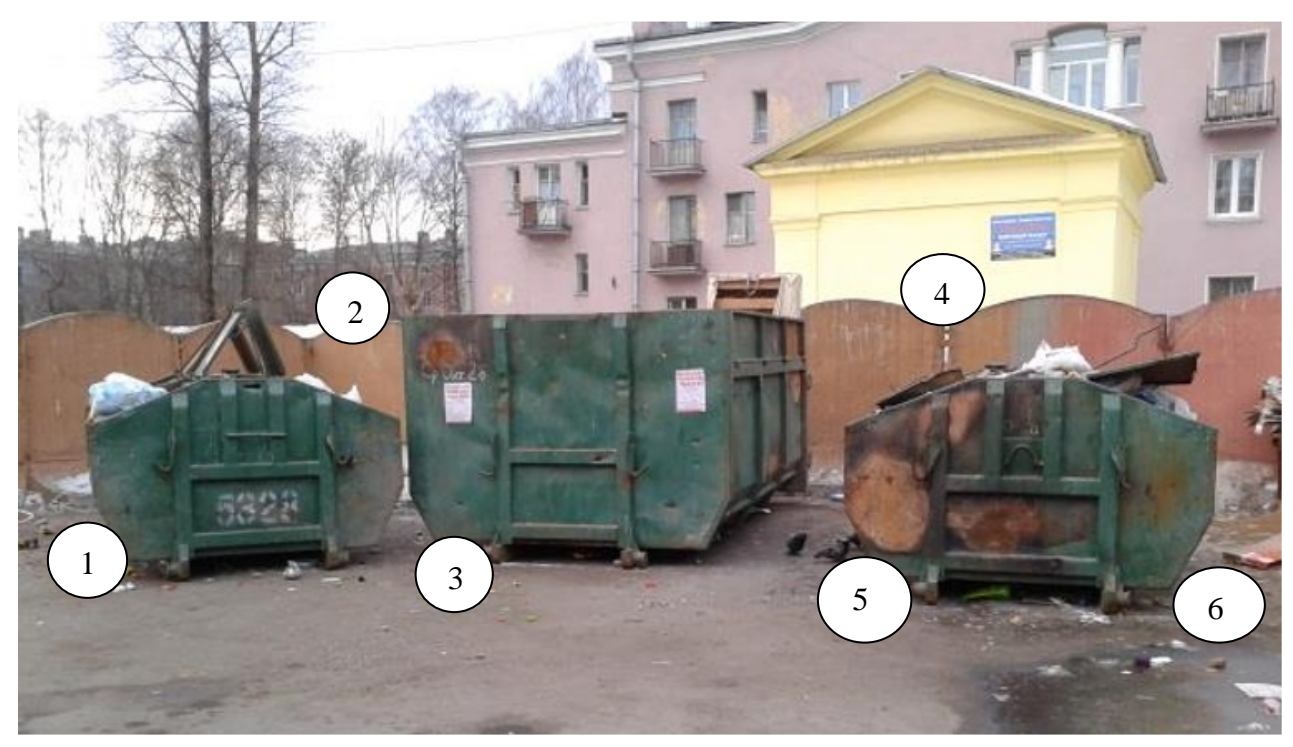

Fig. 1. Air sampling points Krasnogvardeisky district of St. Petersburg.

Table 15. The content of microorganisms in the air of MSW collection sites

\begin{tabular}{|l|c|c|}
\hline \multirow{2}{*}{$\begin{array}{c}\text { Location } \\
\text { (district of St. Petersburg })\end{array}$} & The number of colony forming units \\
\cline { 2 - 3 } Vasileostrovky & $(1,38 \pm 0,12) \times 10^{4}$ & $(3,52 \pm 0,43) \times 10^{3}$ \\
\hline Petrogradsky & $(1,77 \pm 0,18) \times 10^{4}$ & $(4,29 \pm 0,44) \times 10^{3}$ \\
\hline Central & $(1,77 \pm 0,22) \times 10^{4}$ & $(4,29 \pm 0,55) \times 10^{3}$ \\
\hline Admiralteysky & $(1,88 \pm 0,11) \times 10^{4}$ & $(4,16 \pm 0,93) \times 10^{3}$ \\
\hline Seaside & $(1,04 \pm 0,94) \times 10^{4}$ & $(2,77 \pm 0,38) \times 10^{3}$ \\
\hline Vyborg & $(9,45 \pm 0,76) \times 10^{3}$ & $(2,10 \pm 0,30) \times 10^{3}$ \\
\hline Kalininsky & $(7,25 \pm 2,31) \times 10^{3}$ & $(1,94 \pm 0,96) \times 10^{3}$ \\
\hline Krasnogvardeisky & $(4,60 \pm 0,96) \times 10^{3}$ & $(1,11 \pm 0,17) \times 10^{3}$ \\
\hline Nevsky & $(3,25 \pm 0,50) \times 10^{3}$ & $(9,55 \pm 2,45) \times 10^{2}$ \\
\hline Frunze & $(2,60 \pm 0,41) \times 10^{3}$ & $(1,20 \pm 0,26) \times 10^{3}$ \\
\hline Moscow & $(3,05 \pm 0,38) \times 10^{3}$ & $(1,28 \pm 0,18) \times 10^{3}$ \\
\hline Kirovsky & $(3,34 \pm 0,51) \times 10^{3}$ & $(1,25 \pm 0,14) \times 10^{3}$ \\
\hline Krasnoselsky & $(1,57 \pm 0,28) \times 10^{3}$ & $(5,10 \pm 1,34) \times 10^{2}$ \\
\hline
\end{tabular}

Confidence intervals are given for $\mathrm{p}=0.05$ 
Table 16. The influence of MSW processing and disposal facilities on the microbiological composition of atmospheric air

\begin{tabular}{|c|c|c|}
\hline \multirow{2}{*}{$\begin{array}{r}\text { Location } \\
\text { (district of St. Petersburg) }\end{array}$} & \multicolumn{2}{|c|}{ The number of colony forming units } \\
\hline & Bacteria & Micromycetes \\
\hline \multicolumn{3}{|l|}{ Landfill MSW South } \\
\hline summer & $(6,94 \pm 0,65) \times 10^{3}$ & $(2,67 \pm 0,31) \times 10^{3}$ \\
\hline winter & $(6,28 \pm 1,23) \times 10^{2}$ & $(2,17 \pm 0,45) 10^{2}$ \\
\hline \multicolumn{3}{|c|}{$\begin{array}{l}\text { Mechanized Household Waste Processing Experimental Plant } \\
\left(59^{\circ} 48^{\prime} 02.1^{\prime \prime N} 30^{\circ} 08^{\prime} 14.2^{\prime \prime E}\right)\end{array}$} \\
\hline summer & $(2,41 \pm 0,38) \times 10^{3}$ & $(1,35 \pm 0,15) \times 10^{3}$ \\
\hline \multicolumn{3}{|c|}{$\begin{array}{l}\text { Residential buildings closest to these properties } \\
\left(59^{\circ} 47^{\prime} 41.2^{\prime \prime} \mathrm{N} 30^{\circ} 09^{\prime} 14.9^{\prime \prime} \mathrm{E}\right)\end{array}$} \\
\hline summer & $(1,65 \pm 0,34) \times 10^{3}$ & $(4,58 \pm 1,29) \times 10^{2}$ \\
\hline winter & $(6,50 \pm 1,07) \times 10^{2}$ & $(1,96 \pm 0,54) \times 10^{2}$ \\
\hline
\end{tabular}

Confidence intervals are given for $\mathrm{p}=0.05$

\section{Conclusions}

1. MSW collected by the shaft are hazardous for waste sorting personnel before manual sorting; it is necessary to separate the "food waste", "screening" and "other" fractions using a separator.

2. The content of microorganisms in the waste increases during the stay in the container and during transportation in containers to the place of sorting and / or processing.

3. Separate collection of air-dry MSW allows to reduce the microflora content in problem fractions by 5-8 orders of magnitude.

4. The MSW collection sites do not significantly affect the microbiological pollution of atmospheric air.

5. MSW landfills have a significant impact on microbiological pollution of the atmosphere.

\section{References}

1. G. Kozlov, N. Vasilyeva, I. Riabinin, 9th Trends in Medical Mycology 5(95), 241-242 (Nice, France 2019); doi:10.3390/jof5040095.

2 F. Sebők, C. Dobolyi, J Bobvos, et al. Aerobiologia 32(2), 255-263 (2016).

3 C. O. Onwosi, V. C. Igbokwe, J. N. Odimba, et al. Journal of Environmental Management 190, 140-157 (2017).

4. A.V. Musatov, D.S. Yakshilov, O.V. Ischenko, et al., Abstracts of International Conference "Renewable Wood and Plant Resources: Chemistry, Technology, Pharmacology, Medicine”, 144 (SPb.-Repino 2011). 